\title{
Imaging magnetic focusing of coherent electron waves
}

\author{
KATHERINE E. AIDALA ${ }^{1 *}$, ROBERT E. PARROT² ${ }^{2}$ TOBIAS KRAMER², E. J. HELLER ${ }^{2,3}$, \\ R. M. WESTERVELT ${ }^{1,2 \dagger}$, M. P. HANSON ${ }^{4}$ AND A. C. GOSSARD ${ }^{4}$ \\ ${ }^{1}$ Div. of Engineering and Applied Sciences, Harvard University, Cambridge, Massachusetts 02138, USA \\ ${ }^{2}$ Dept. of Physics, Harvard University, Cambridge, Massachusetts 02138, USA \\ ${ }^{3}$ Dept. of Chemistry and Chemical Biology, Harvard University, Cambridge, Massachusetts 02138, USA \\ ${ }^{4}$ Dept. of Materials Science, University of California, Santa Barbara, California 93106, USA \\ *Present address: Dept. of Physics, Mount Holyoke College, South Hadley, Massachusetts 01075, USA \\ †e-mail: westervelt@deas.harvard.edu
}

The coherent flow of electrons through a two-dimensional electron gas $^{1-8}$ (2DEG) offers promising approaches for spintronics $^{8-10}$ and quantum information processing ${ }^{11,12}$. Cryogenic scanning probe microscopes (SPMs) are a valuable tool for imaging electron motion ${ }^{13-25}$, but have been limited by their inability to follow such motion through an open structure under an applied magnetic field. Here we report a way to visualize the flow of electron waves from one point to another by using the SPM tip to create a lens in the 2DEG below. The lens deflects electrons and casts a shadow downstream. We use this technique to image magnetic focusing in a GaAs 2DEG. Magnetic focusing occurs when electrons flowing from one quantum point contact (QPC) rejoin at a second QPC a number of cyclotron diameters away $^{3,4,9,26,27}$. Our images show semicircular trajectories as the electrons bounce along the boundary, as well as fringes created by the interference of multiple paths, demonstrating that the flow is coherent ${ }^{18,19,28}$. Remarkable agreement between experiment and theory demonstrates our ability to visualize electron trajectories in a magnetic field, and to make a new type of imaging electron interferometer.

The coherent motion of electrons through an open device ${ }^{1-8}$ in a magnetic field offers promising approaches to spintronics ${ }^{8-10}$ and quantum information processing ${ }^{11,12}$. Electron spins can travel coherently over distances greater than $100 \mu \mathrm{m}$ through a two-dimensional electron gas (2DEG) in $\mathrm{GaAs}^{8}$. The spin-orbit interaction couples the electron spin with its orbital motion. As a result, the spin direction can change the cyclotron radius, as observed in splitting of magnetic focusing peaks in conductance for a two-dimensional hole gas ${ }^{9}$. The spin-orbit interaction is also predicted $^{10}$ to produce in semiconductors an analogy of Zitterbewegung for relativistic systems-the direction of the spin oscillates sideways as an electron moves through the 2DEG. For quantum information processing, it is proposed ${ }^{12}$ that the entanglement of two electron spins can be detected through the angular dependence of their collision cross-section. To explore these new directions, one needs to track the motion of electrons in a magnetic field.

A cooled scanning probe microscope (SPM) can image electrons in a 2DEG below by using the tip as a movable gate ${ }^{13-25}$. Pathways for electron waves emerging from a quantum point contact (QPC) were revealed ${ }^{18}$ and found to have a dramatic branched form ${ }^{19}$ due to small-angle scattering - the well-known process that limits the mobility. A charged tip was able to image this flow by fully depleting the 2DEG below to form a divot that backscattered electrons arriving from the QPC-some follow a time-reversed path back through the QPC, reducing its conductance. Flow from the QPC was imaged by displaying the drop in conductance as the tip was scanned above ${ }^{18,19}$. However, this technique does not work in a magnetic field, when the orbits bend. The electrons can no longer return to the QPC along a timereversed path, and the measured image intensity falls dramatically. A new technique must be developed, which can image flow from one point to a different location.

In this letter, we demonstrate a new way to image the flow of electron waves between two points in an open device using a cooled SPM, and we demonstrate this approach by imaging magnetic focusing of electron waves in a 2DEG. Magnetic focusing plays an important role in ballistic transport ${ }^{3,4,9,26,27}$ - electrons flowing away from one QPC at different angles follow cyclotron orbits of the same radius $r_{\mathrm{c}}$ and rejoin at a second QPC located a number of diameters $2 r_{\mathrm{c}}$ away. To image flow in a magnetic field, we used a new technique in which the SPM tip partially depletes the 2DEG to create a lens that redirects flow, throwing a shadow downstream. We obtain clear pictures of the semicircular bouncing-ball patterns of flow that cause magnetic focusing. Branching of the flow is visible. In addition, we see fringes created by the interference of electron waves bouncing off the tip with those travelling directly between the two QPCs. In this way, the cooled SPM acts as a new type of imaging electron interferometer, in addition to our earlier device, which uses an electron mirror ${ }^{28}$. The observed bouncing-ball patterns of flow and interference fringes are in excellent agreement with full quantum simulations that include the tip-induced electron lens and smallangle scattering, demonstrating that this new imaging technique accurately images flow of coherent electron waves through a $2 \mathrm{DEG}$ in a magnetic field.

Figure 1 illustrates the device geometry and the imaging technique. Two QPCs are formed by surface gates (Fig. 1a,b); the QPC centres are separated by $L=2.7 \mu \mathrm{m}$. A 2DEG with density $3.8 \times 10^{11} \mathrm{~cm}^{-2}$ and mobility $500,000 \mathrm{~cm}^{2} \mathrm{~V}^{-1} \mathrm{~s}^{-1}$ is located $47 \mathrm{~nm}$ 


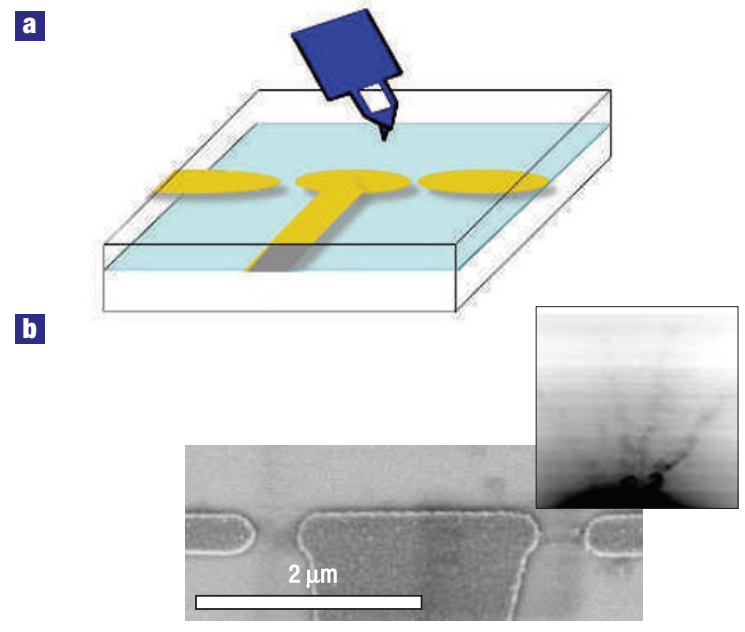

$\mathbf{G}$

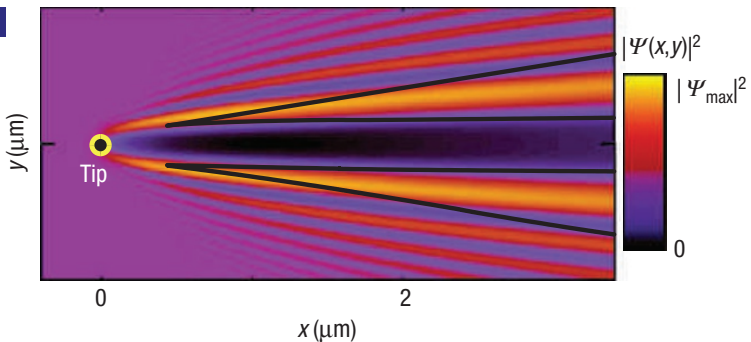

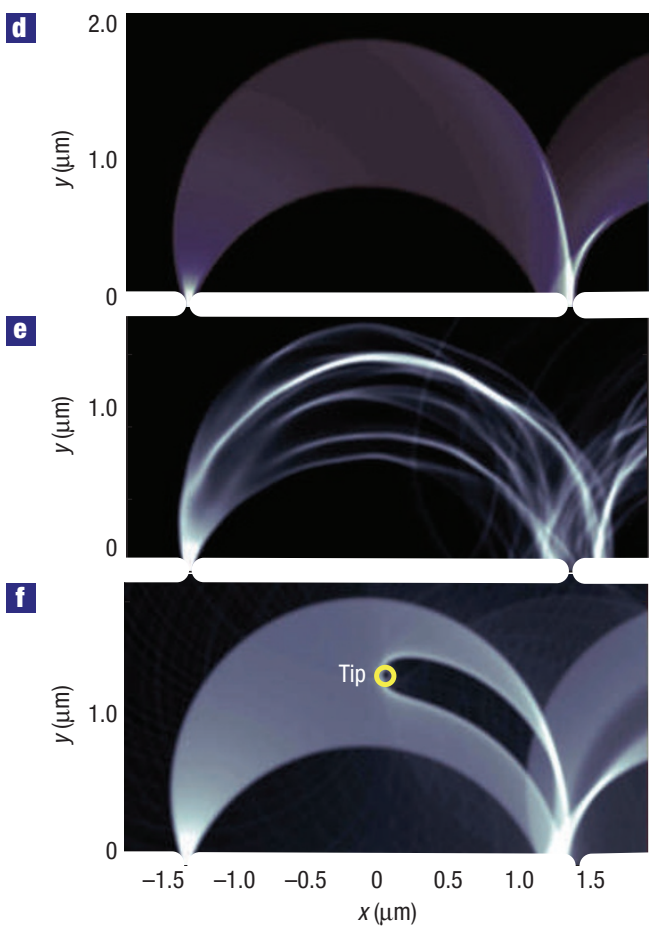

Figure 1 Set-up for SPM images and simulations of magnetic focusing in a 2DEG. a, Schematic diagram showing a conducting SPM tip scanned above the surface of a device. $\mathbf{b}$, Scanning electron micrograph of the magnetic focusing device; the inset shows an SPM image of the electron flow at zero magnetic field, displaying branches in electron flow. c, Quantum simulation showing the scattering of an incoming plane wave by an SPM tip, with 2DEG Fermi energy $E_{\mathrm{F}}=13 \mathrm{meV}$, and a gaussian tip potential with height $0.2 E_{\mathrm{F}}$ and width $50 \mathrm{~nm}$. d-f, Classical simulations of magnetic focusing in a magnetic field: in a flat potential, showing circular cyclotron orbits (d), with small-angle scattering, showing added branches (e), and in a flat potential with an SPM tip (f).

beneath the surface of a GaAs/AlGaAs heterostructure with a $5 \mathrm{~nm}$ GaAs cap, a $20 \mathrm{~nm} \mathrm{Al}_{0.3} \mathrm{Ga}_{0.7}$ As barrier and a Si delta-doping layer, then a $22 \mathrm{~nm} \mathrm{Al}_{0.3} \mathrm{Ga}_{0.7}$ As barrier next to the 2DEG in GaAs. Metal QPC gates were fabricated using e-beam lithography. The device is mounted in the SPM, inside a superconducting solenoid, and cooled to $4.2 \mathrm{~K}$. A computer controls the SPM and records the images.

Magnetic focusing (Fig. 1d) occurs because electrons leaving one QPC over a range of angles circle around and rejoin at the second QPC, when the spacing $L$ is close to the diameter of a cyclotron orbit. For GaAs the electron cyclotron orbit is circular, with radius

$$
r_{\mathrm{c}}=\hbar k_{\mathrm{F}} / e B
$$

where $e$ is the electron charge, $B$ is the perpendicular magnetic field and $k_{\mathrm{F}}$ is the Fermi wavevector. As $B$ is increased, the first focusing peak occurs when $L=2 r_{\text {c }}$. Additional peaks occur at higher fields when $L=2 n r_{\mathrm{c}}$ is an integer multiple of the cyclotron diameter at fields

$$
B_{n}=2 n \hbar k_{\mathrm{F}} / e L
$$

The shape, clarity and spacing of magnetic focusing peaks provide information about ballistic flow in the sample material. The effects of small-angle scattering are shown in the zero-field image in Fig. 1b, the simulation of Fig. 1e and the movie in the Supplementary Information. Magnetic focusing occurs, but the flow now contains branches ${ }^{19}$ similar to those in Fig. 1 b.
To image electron flow from one point to another, a small movable electron lens is created by a dip in electron density immediately below the charged SPM tip. The lens deflects electrons (Fig. 1c), throwing a V-shaped shadow downstream. An image of electron flow is obtained by displaying the change in transmission $\Delta T$ between the QPCs as the tip is scanned across a plane $10 \mathrm{~nm}$ above the surface. The transmission $T$ is proportional to the voltage across the second QPC when a known current is passed through the first. When electrons are ballistically injected into the second QPC from the first, a voltage develops, which drives a current in the opposite direction, which cancels the influx of electrons.

Simulations of electron flow that show how the imaging technique works are presented in Fig. 1c-f. A gaussian $\varphi_{\text {tip }}=V_{0} \exp \left(-\left(r-r_{\text {tip }}\right)^{2} / 2 a^{2}\right)$ is used to model the tip potential in the 2DEG, where $r_{\text {tip }}$ is the tip position, $a$ is the width and $V_{0}$ is the height. For this paper $V_{0}>0$, and the tip creates a dip in electron density. The relative strength of the tip potential is

$$
\eta=\frac{V_{0}}{E_{\mathrm{F}}},
$$

where $E_{\mathrm{F}}$ is the Fermi energy. For $\eta<1$, the electron gas is partially depleted, and an imperfect diverging lens is created with a focal length determined by $\eta$. When $\eta \geq 1$, the 2DEG is fully depleted, and electrons can backscatter. Simulations (Fig. 1c) at $B=0$ show how a weak lens $(\eta=0.2)$ creates a $\mathrm{V}$-shaped shadow downstream by forcing electrons to the sides, where they form two caustics in flow along the legs of the V. Figure if shows how 

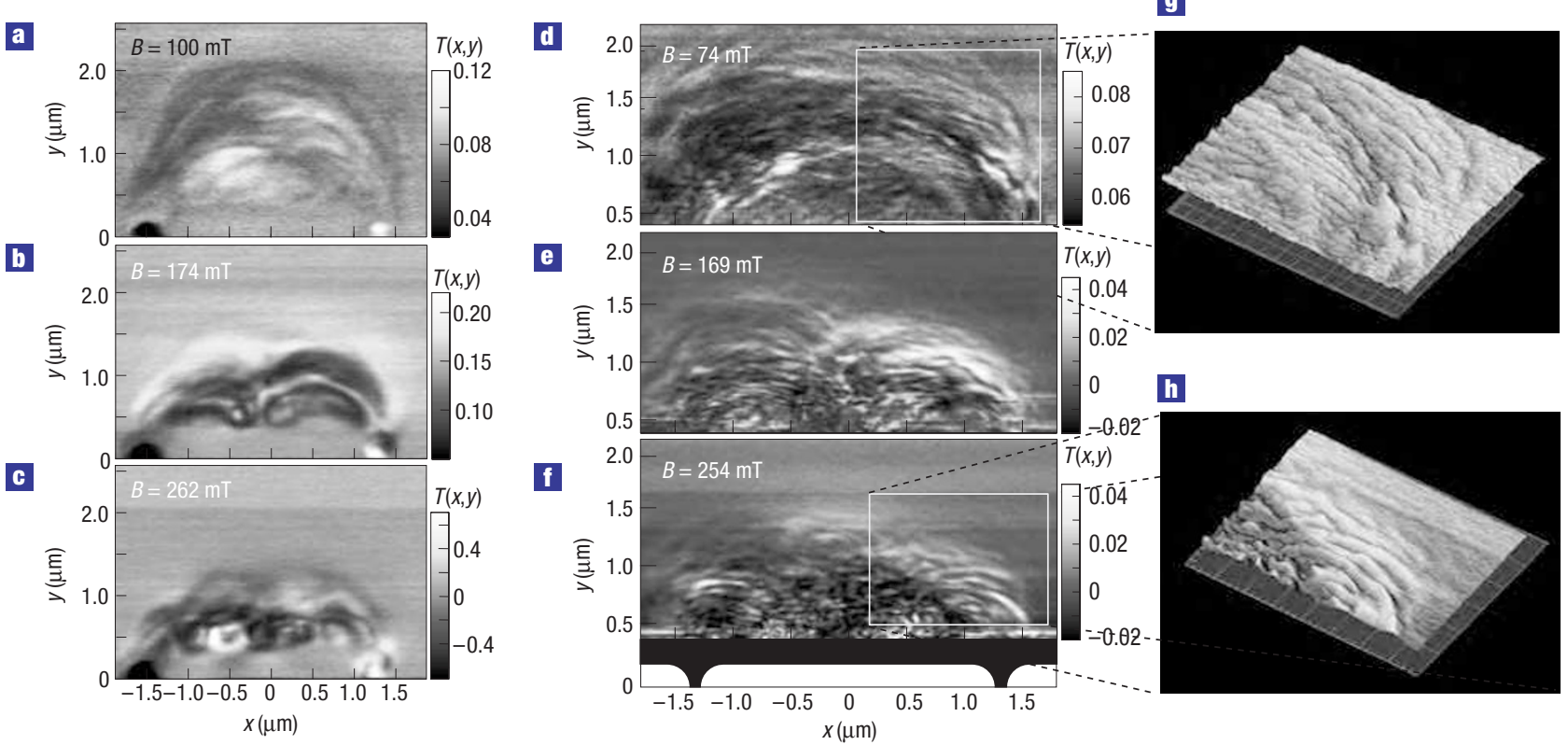

Figure 2 Experimental SPM images of magnetic focusing in a $2 D E G$ at $4.2 \mathrm{~K}$ recorded near the first three magnetic focusing peaks. a-c, A weakly focusing $(\eta \approx 0.5)$ tip $90 \mathrm{~nm}$ above the 2DEG recorded at $B=100 \mathrm{mT}, B=174 \mathrm{mT}$ and $B=262 \mathrm{mT}$ with cyclotron radii $r_{\mathrm{c}}=970 \mathrm{~nm}, r_{\mathrm{c}}=560 \mathrm{~nm}$ and $r_{\mathrm{c}}=370 \mathrm{~nm}$, respectively. The left QPC is on the first conductance plateau, the right is on the third. $\mathbf{d}-\mathbf{f}$, Strongly focusing $(\eta \approx 1.0)$ tip $60 \mathrm{~nm}$ above the 2DEG recorded at $B=74 \mathrm{mT}, B=169 \mathrm{mT}$ and $B=254 \mathrm{mT}$ with cyclotron radii $r_{\mathrm{c}}=1310 \mathrm{~nm}, r_{\mathrm{c}}=580 \mathrm{~nm}$ and $r_{\mathrm{c}}=380 \mathrm{~nm}$, respectively. Both QPCs are on the second conductance plateau. The grey scale shows the change in transmission between the QPCs induced by the tip. $\mathbf{g}, \mathbf{h}$, Close-up surface plots in the yellow rectangles of $\mathbf{d}$ and $\mathbf{f}$ that show the regularity and consistency of the quantum fringe structure.
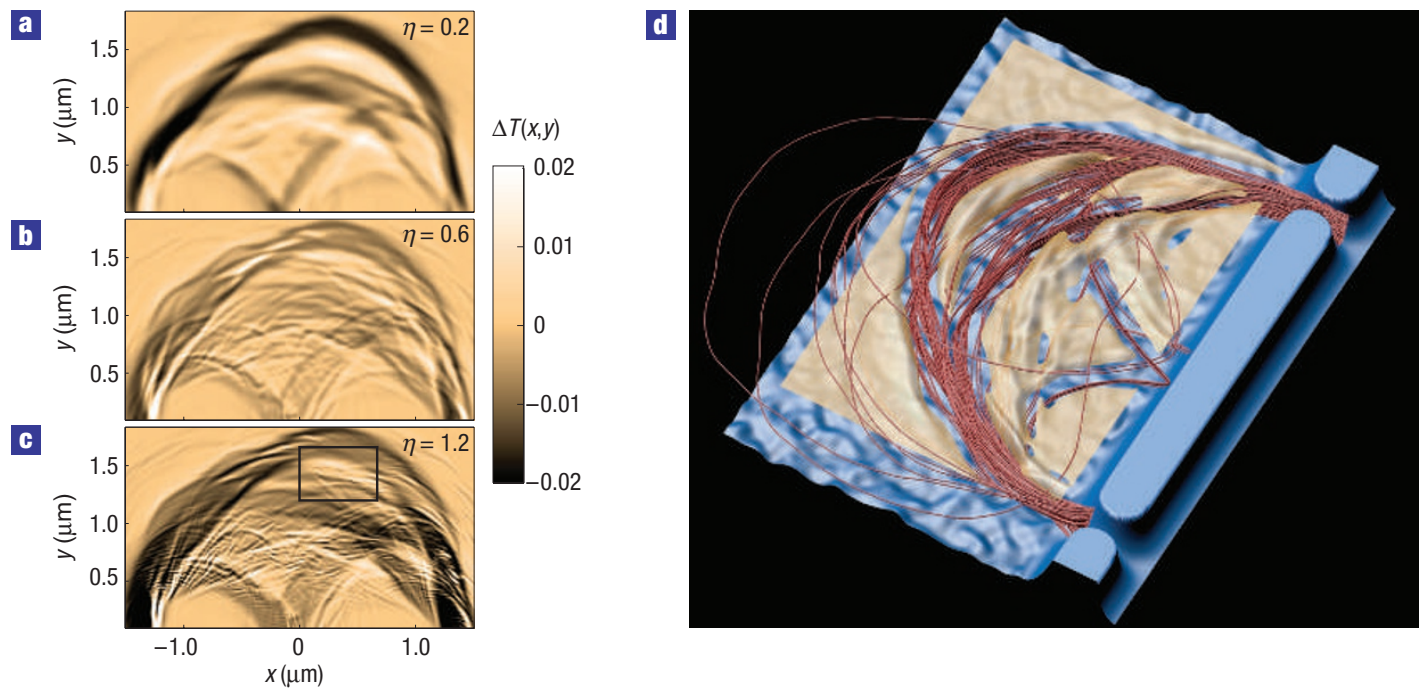

Figure 3 Quantum simulations of SPM images including small-angle scattering. The panels display the change in transmission $\Delta T$ between QPCs versus tip position on the first magnetic focusing peak $(B=77 \mathrm{mT})$ for $\lambda_{\mathrm{F}}=40 \mathrm{~nm}$ at $1.7 \mathrm{~K}$. a, For a weak tip $(\eta=0.2)$ that scatters into small angles, the dark area of reduced transmission $(\Delta T<0)$ shows a classical cyclotron orbit. $\mathbf{b}, \mathbf{c}$, , For moderate $(\eta=0.6)(\mathbf{b})$ and strong $(\eta=1.2)(\mathbf{c})$ tips, quantum interference fringes become visible. $\mathbf{d}$, Correspondence between the simulated SPM image (beige surface) from a and ray-tracing calculations of the originally transmitted electron trajectories (red lines) before the tip was present; regions with $\Delta T<0$ are omitted for ease of comparison. The blue surface is the smoothly disordered background potential. The dark areas with reduced transmission $(\Delta T<0)$ line up very well with the original classical trajectories. For stronger tips, imaging of the original classical trajectories becomes more difficult, but fringing reveals regions of high coherent flux of electron waves.

we image magnetic focusing: when the shadow cast by the lens beneath the tip hits the second QPC, $T$ is reduced. The amount of reduction $\Delta T$ is proportional to the original flux before the tip was introduced. By displaying $\Delta T$ versus tip position $r_{\text {tip }}$ as the tip is raster scanned in a plane above, an image of the original flow is created ${ }^{29}$ 


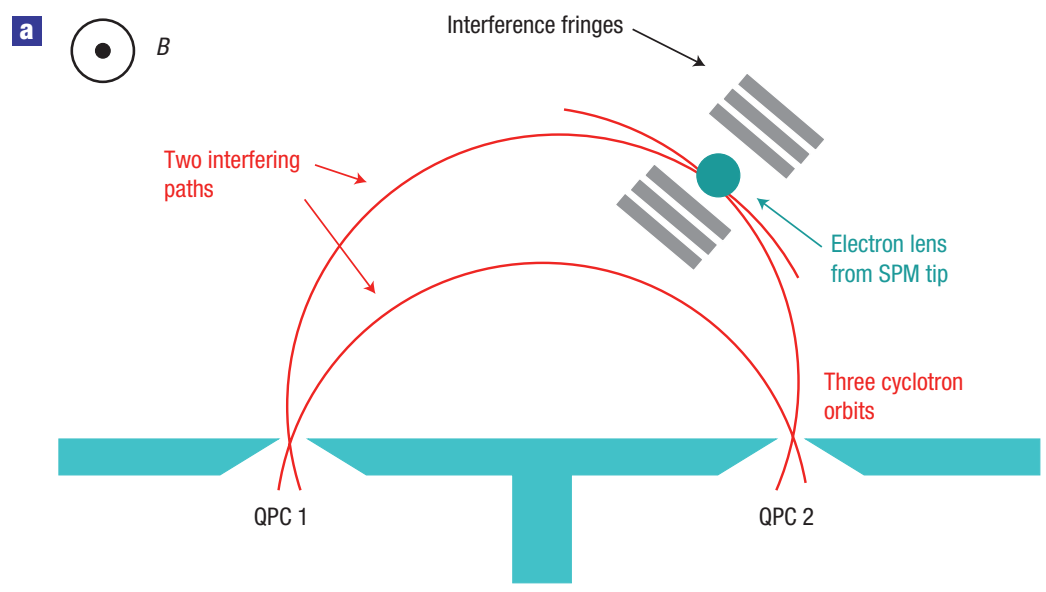

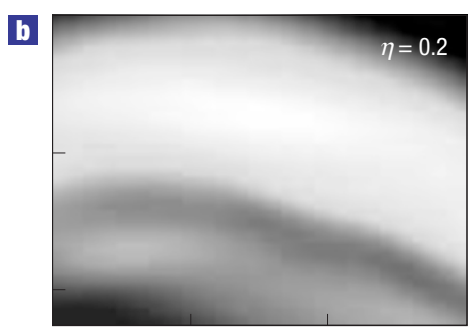

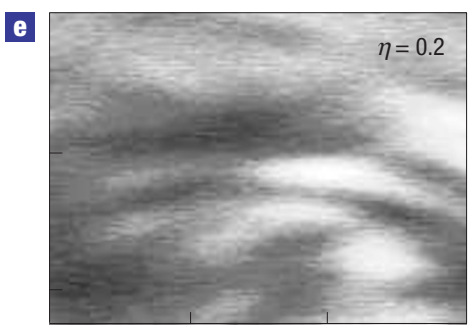

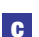
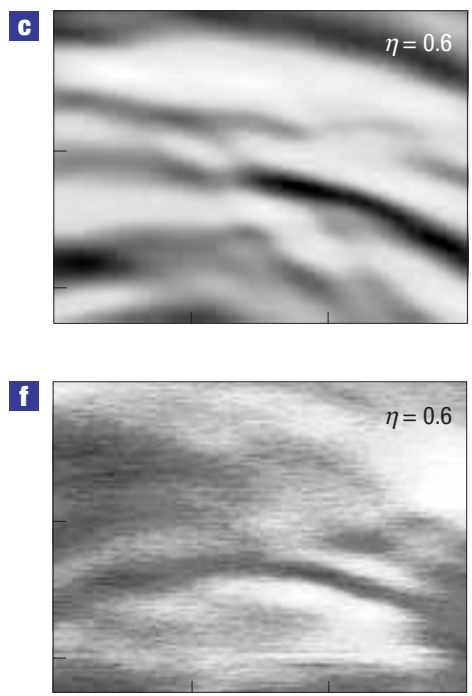

d

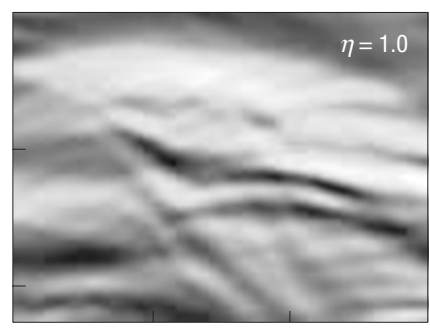

g

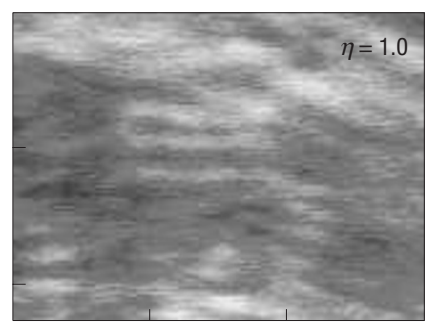

Figure 4 Direct comparison of interference fringes between experiment and theory for different tip strengths. a, How interference occurs between a path deflected by the SPM tip and a direct path between the two QPCs. b-d, Quantum simulations of interference fringes at $1.7 \mathrm{~K}$ and $B=77 \mathrm{~T}$ for weak $(\eta=0.2)$, moderate $(\eta=0.6)$ and strong $(\eta=1.0)$ tip strengths; $\lambda_{\mathrm{F}}=40 \mathrm{~nm}$. The panels are located in the black box in Fig. 3c. e-g, SPM images at $B=173 \mathrm{~T}$ showing fringes that appear and move closer as the tip strength $\eta$ increases, in good agreement with the simulations. The images have dimensions $600 \times 450 \mathrm{~nm}^{2}$ and are located $750 \mathrm{~nm}$ above and $500 \mathrm{~nm}$ to the right of the midpoint between the two QPCs.

Experimental images of magnetic focusing near the first, second and third peaks are presented in Fig. $2 \mathrm{a}-\mathrm{c}$ for a weak tip $(\eta \approx 0.5)$ and in Fig. $2 \mathrm{~d}-\mathrm{f}$ for a strong tip $(\eta \approx 1.0)$. The bouncing semicircular cyclotron orbits imaged here are an experimental visualization of the origins of magnetic focusing. Figure 2a shows a single, semicircular crescent characteristic of the first peak. Branches in flow from small-angle scattering are also visible. For Fig. $2 \mathrm{~b}$ the bounce characteristic of the second peak is clearly seen. For Fig. 2c, recorded near the third focusing peak, it becomes difficult to see distinct bounces, although circular features are evident, with radii comparable to $r_{\mathrm{c}}$. The bouncing-ball orbits begin to form the semi-classical equivalent of an edge state.

With a strongly scattering tip $(\eta \sim 1.0)$ the SPM images (Fig. 2d-f) have new and distinctly finer features, resulting from the interference of electron waves, some travelling along new pathways created by scattering at the tip. In the absence of the tip, electrons move from one QPC to the other by simultaneously travelling along multiple paths, which add up with a particular overall phase. As quantified below, a strongly scattering tip introduces new trajectories (and removes some of the old ones). These new trajectories interfere with the original ones, and create interference fringes as the tip is scanned, which can be seen in the experimental images. The images in Fig. $2 \mathrm{~d}-\mathrm{f}$ also show bouncing-ball orbits similar to their counterparts in Fig. 2a-c. The striking difference is the appearance of narrow fringelike features. In some locations, a noticeably periodic structure of fringes exists, shown in the blowups, Fig. 2g,h.

We can understand both the classical and quantum behaviour by using full, thermally averaged quantum simulations of an SPM image including tip scattering. Figure $3 \mathrm{a}-\mathrm{c}$ shows simulations of an image on the first magnetic focusing peak for weak $(\eta=0.2)$, moderate $(\eta=0.6)$ and strong $(\eta=1.2)$ tips. These were obtained at $1.7 \mathrm{~K}$ using a thermal wavepacket calculation ${ }^{30}$ with Fermi wavelength $\lambda_{\mathrm{F}}=40 \mathrm{~nm}$, and they include a random background potential from donor ions. For the weak tip in Fig. 3a, the dark area of reduced transmission $(\Delta T<0)$ corresponds to a classical 
cyclotron orbit. For the moderate and strong tips in Fig. 3b,c, quantum interference fringes created by tip scattering become visible. The increase in fringelike structure as the tip strength is increased is in excellent qualitative agreement with the behaviour of the experimental SPM images in Fig. 2a-c and Fig. 2d-f.

To understand the source of contrast in the experimental images, it is very useful to compare quantum simulations with classical trajectories ${ }^{18,19}$. Figure $3 d$ joins quantum simulations from Fig. 3a (beige surface) with classical trajectories (red lines) computed without the tip present using ray tracing. The background potential is shown in blue. Areas with $\Delta T<0$ are eliminated for ease of comparison. We see that the original trajectories line up with paths of decreased transmission, because the flow is blocked by the tip. These simulations reveal the unusual, and yet very informative, way that electron flow is encoded in the experimental images.

The origin of fringing can be understood by a simple semiclassical argument pictured in Fig. 4a. When a new trajectory with phase $\phi$ deflects from the tip and reaches the target QPC, it contributes an amplitude $a \mathrm{e}^{i \phi}$ which interferes coherently with the background amplitude $A_{0} \mathrm{e}^{i \phi_{0}}$ from the nascent trajectories, such that the change in transmission depends on the phase difference $\Delta T \propto \cos \left(\phi-\phi_{0}\right)$. This is illustrated in Fig. 4a by the interference of a direct path between the QPCs along a single cyclotron orbit with a path deflected by the tip, composed of two cyclotron orbit segments. The phase of the deflected trajectory $\phi=S / \hbar$ is proportional to the classical action $S$ accumulated along the trajectory. When the tip is moved, $S$ changes, and so does $\phi$. This leads to a simple equation for the fringe spacing $d$ :

$$
d=\frac{\lambda_{\mathrm{F}}}{2} \operatorname{cosec} \frac{\theta}{2},
$$

where $\theta$ is the angle by which the trajectory has been deflected by the tip, shown in Fig. 4a. When the tip backscatters by $\theta=\pi$, the fringe spacing is $\lambda_{\mathrm{F}} / 2$, as has been seen in previous experiments ${ }^{18,19}$. When using a weak tip with $\eta<1$, the maximum possible scattering angle is $\theta<\pi$, because it cannot backscatter. This implies that the minimum fringe spacing is $d_{\min }>\lambda_{\mathrm{F}} / 2$. For sufficiently weak tips $\eta \sim 0.1$, the fringe spacings are so large that they are indistinguishable from classical structures such as branches.

A direct comparison of fringing between theory (Fig. 4b-d) and experiment (Fig. 4e-g) shows remarkable agreement. A series of quantum simulations in a small region (outlined in black in Fig. 3c) is compared with SPM images of a comparable area. The simulations in Fig. $4 \mathrm{~b}-\mathrm{d}$ are for $\eta=0.2,0.6$ and 1.0 respectively, with the corresponding experimental images for these tip strengths displayed below in Fig. 4e-g. The agreement is excellent. The fringe spacing for a strong tip is comparable to $\lambda_{\mathrm{F}}$, to be expected when the electrons are scattered by $\theta \sim \pi / 3$. In this geometry the SPM acts as an interferometer that could be used to extract information from the fringes about the momentum and energy of the electrons.

Received 7 January 2007; accepted 25 April 2007; published 3 June 2007.
References

1. Sohn, L. L., Kouwenhoven, L. P. \& Schon, G. Mesoscopic Electron Transport (Kluwer Academic, 1997)

2. van Wees, B. J. et al. Quantized conductance of point contacts in a two-dimensional electron gas. Phys. Rev. Lett. 60, 848-850 (1988).

3. van Houten, H. et al. Coherent electron focusing with quantum point contacts in a two-dimensional electron gas. Phys. Rev. B 39, 8556-8575 (1989).

4. Beenakker, C. W. M. \& van Houten, H. Billiard model of a ballistic multiprobe conductor. Phys. Rev Lett. 63, 1857-1860 (1989).

5. Shepard, K. L., Roukes, M. L. \& Van der Gaag, B. P. Direct measurement of the transmission matrix of a mesoscopic conductor. Phys. Rev. Lett. 68, 2660-2663 (1992).

6. Crommie, M. F., Lutz, C. P. \& Eigler, D. M. Confinement of electrons to quantum corrals on a metal surface. Science 262, 218-220 (1993).

7. Katine, J. A. et al. Point contact conductance of an open resonator. Phys. Rev. Lett. 79, 4806-4809 (1997).

8. Kikkawa, J. M. \& Awschalom, D. D. Lateral drag of spin coherence in gallium arsenide. Nature 397, 139-141 (1999).

9. Rokhinson, L. P., Larkina, V., Lyanda-Geller, Y. B., Pfeiffer, L. N. \& West, K. W. Spin separation in cyclotron motion. Phys. Rev. Lett. 93, 146601 (2004).

10. Schliemann, J., Loss, D. \& Westervelt, R. M. Zitterbewegung of electronic wave packets in III-V zinc-blende semiconductor quantum wells. Phys. Rev. Lett. 94, 206801 (2005).

11. Awschalom, D. D., Loss, D. \& Samarth, N. Semiconductor Spintronics and Quantum Computation (Springer, Berlin, 2002)

12. Saraga, D. S., Altshuler, B. L., Westervelt, R. M. \& Loss, D. Coulomb scattering in a 2 D interacting electron gas and production of EPR pairs. Phys. Rev. Lett. 92, 246803 (2004).

13. Topinka, M. A., Westervelt, R. M. \& Heller, E. J. Imaging electron flow. Phys. Today 56, 47-52 (2003).

14. Eriksson, M. A. et al. Cryogenic scanning-probe characterization of semiconductor nanostructures. Appl. Phys. Lett. 69, 671-673 (1996)

15. Tessmer, S. H., Glicofridis, P. I., Ashoori, R. C., Levitov, L. S. \& Melloch, M. R. Subsurface charge accumulation imaging of a quantum Hall liquid. Nature 392, 51-54 (1998).

16. Yacoby, A., Hess, H. F., Fulton, T. A., Pfeiffer, L. N. \& West, K. W. Electrical imaging of the quantum Hall state. Solid State Commun. 111, 1-13 (1999).

17. McCormick, K. L. et al. Scanned potential microscopy of edge and bulk currents in the quantum Hall regime. Phys. Rev. B 59, 4654-4657 (1999)

18. Topinka, M. A. et al. Imaging coherent flow from a quantum point contact. Science 289, 2323-2326 (2000).

19. Topinka, M. A. et al. Coherent branched flow in a two-dimensional electron gas. Nature $\mathbf{4 1 0}$, 183-188 (2001).

20. Steele, G. A., Ashoori, R. C., Pfeiffer, L. N. \& West, K. W. Imaging transport resonances in the quantum Hall effect. Phys. Rev. Lett. 95, 136804 (2005).

21. Crook, R., Smith, C. G., Simmons, M. Y. \& Ritchie, D. A. Imaging cyclotron orbits and scattering sites in a high-mobility two-dimensional electron gas. Phys. Rev. B 62, 5174-5178 (2000)

22. Crook, R. et al. Erasable electrostatic lithography for quantum computers. Nature $\mathbf{4 2 4}$ 751-755 (2003).

23. Ihn, T. et al. Local spectroscopy of edge channels in the quantum Hall regime with local probe techniques. Physica E 13, 671-674 (2002).

24. Kicin, S. et al. Local backscattering in the quantum Hall regime. Phys Rev. B 70, 205302 (2004).

25. Aoki, N., da Cunha, C. R., Akis, R., Ferry, D. K. \& Ochiai, Y. Imaging of integer quantum Hall edge state in a quantum point contact via scanning gate microscopy. Phys. Rev. B 72, 155327 (2005).

26. Sharvin, Y. V. \& Fisher, L. M. Observation of focused electron beams in a metal. JETP Lett. 1, 152-153 (1965).

27. Tsoi, V. Focusing of electrons in a metal by a transverse magnetic field. JETP Lett. 19, 70-71 (1974)

28. LeRoy, B. J. et al. Imaging electron interferometer. Phys. Rev. Lett. 94, 126801 (2005).

29. Aidala, K. E., Parrott, R. E., Heller, E. J. \& Westervelt, R. M. Imaging electrons in a magnetic field. Physica E 34, 409-412 (2006).

30. Heller, E. J. et al. Thermal averages in a quantum point contact with a single coherent wave packet. Nano Lett. 5, 1285-1292 (2005).

\section{Acknowledgements}

This work has been performed with support at Harvard University from the ARO, the NSF-funded Nanoscale Science and Engineering Center (NSEC), and the DFG (Emmy-Noether program). Work at Santa Barbara has been supported in part by the Institute for Quantum Engineering, Science and Technology (iQUEST). We would also like to thank the National Nanotechnology Infrastructure Network (NNIN) and the Harvard CrimsonGrid for computing resources.

Correspondence and requests for materials should be addressed to R.M.W.

Supplementary Information accompanies this paper on www.nature.com/naturephysics.

\section{Author contributions}

K.E.A. conducted the experiments with R.M.W.; R.E.P. and T.K. carried out classical and quantum simulations of electron flow with E.J.H. and M.P.H. grew the semiconductor heterostructure with A.C.G.

\section{Competing financial interests}

The authors declare no competing financial interests.

Reprints and permission information is available online at http://npg.nature.com/reprintsandpermissions/ 